\title{
Basophils priming in patients with chronic spontaneous urticaria
}

\author{
Krzysztof Gomułka, Marta Wrześniak, Wojciech Mędrala, Bernard Panaszek
}

Department of Internal Medicine, Pneumology and Allergology, Medical University of Wroclaw, Wroclaw, Poland

Adv Dermatol Allergol 2021; XXXVIII (4): 608-610

DOI: https://doi.org/10.5114/ada.2021.108910

\begin{abstract}
Introduction: Basophils are one of the main target cells in chronic spontaneous urticaria (CSU). If cells present higher susceptibility to production and degranulation of pro-inflammatory factors, priming may be associated with severity of symptoms and therapy ineffectiveness.

Aim: To evaluate the spontaneous state of increase in basophil activity and their priming profile in patients with CSU.

Material and methods: The study sample included 22 patients diagnosed with CSU and 20 healthy volunteers without either allergy symptoms or CSU. In this study, we evaluate the presence of CD63 and CD63+CD203C at basophils surface by flow cytometry test (basophil activation test - BAT).

Results: We found that the percentage of activated basophils was higher in patients with CSU than in the control group and this difference was statistically significant $(p<0.05)$.

Conclusions: Our results indicate a greater degree of basophils activation in patients with CSU in remission than in the control group; it might be useful for identification of patients with predominance of the autoimmune variant of CSU and typing patients responding (responders) and refractory (non-responders) to treatment with antihistamines.

Key words: basophil, CD203c, CD63, chronic spontaneous urticaria.
\end{abstract}

\section{Introduction}

Mediators released from basophils in IgE-dependent reaction seem to be essential for chronic spontaneous urticaria (CSU). Equally interesting is the process of spontaneous increase of basophil activity (priming) linked with a higher expression of receptors on the cell surface. According to these phenomena, basophils become more sensitive (stand-by) for activation by various factors, outer (allergens, temperature, pressure) and inner (autoreactive immunoglobulin E (IgE) or lgG against self-antigens, IgE and FceRI) [1, 2]. Despite the fact that CSU is not a fully allergic disease, specific priming and basophils sensitization might be higher in patients with CSU than in the global adult population. What is more, IgE might affect autoantigens present in the blood in other autoimmune diseases (e.g. Hashimoto thyroiditis, Sjögren syndrome). A new insight into the pathogenesis of autoimmune diseases points at a higher concentration of autoreactive IgE or IgG as a potential kind of an autoimmune disease, a clinical display of some undefined autoimmune diseases, or both [3-5]. Basophils are one of the main target cells in CSU. If cells present higher susceptibility to production and degranulation of pro-inflammatory factors, priming may be associated with severity of symptoms and therapy ineffectiveness. Reviewing the updated literature, it seems that data on a possible spontaneous increase in basophil activity are lacking.

\section{Aim}

This pilot study aimed to evaluate the spontaneous state of increase in basophil activity and better understanding of their priming profile in patients with CSU.

\section{Material and methods}

Forty-two patients have been included in the study (mean age: 20.5 years; 25 females) of whom 22 patients (mean age: 22 years; 11 females) were diagnosed with CSU and had avoided systemically administered antiallergic drugs at least 14 days prior to blood sampling. A control group of healthy volunteers comprised 20 par-

Address for correspondence: Krzysztof Gomułka PhD, Department of Internal Medicine, Pneumology and Allergology, Medical University of Wroclaw, Wroclaw, Poland, phone: +48 7178426 47, e-mail: kgomulka@wp.pl Received: 23.01.2020, accepted: 12.03.2020. 
Table 1. Basophil activation in the examined groups - CD63 expression

\begin{tabular}{lcccccccccc}
\hline Parameter & \multicolumn{4}{c}{ Patients with CSU } & \multicolumn{5}{c}{ Control group } \\
\cline { 2 - 13 } & PB $^{*}$ & PC1 & PC2 & A1 & A2 $^{\#}$ & PB $^{*}$ & PC1 & PC2 & A1 & A2 $^{*}$ \\
\hline Basophil activity (\%) & 0.351 & 56.155 & 28.374 & 0.334 & 1.774 & $0.0 \pm 0.0$ & 66.381 & 30.527 & 0.062 & 0.0375 \\
Mean \pm SD & \pm 0.612 & \pm 37.049 & \pm 21.851 & \pm 0.604 & \pm 5.281 & & \pm 25.578 & \pm 21.21 & \pm 0.171 & \pm 0.126 \\
\hline Minimum (\%) & 0.0 & 0.24 & 1.18 & 0.0 & 0.0 & 0.0 & 0.0 & 0.41 & 0.0 & 0.0 \\
\hline Maximum (\%) & 2.27 & 97.2 & 77.68 & 2.21 & 22.22 & 0.0 & 88.79 & 64.29 & 0.69 & 0.53 \\
\hline
\end{tabular}

SD - standard deviation, ${ }^{*} p=0.0444,{ }^{*} p=0.0444$.

Table 2. Basophils activation in the examined groups - CD63+CD203c expression

\begin{tabular}{|c|c|c|c|c|c|c|c|c|c|c|}
\hline \multirow[t]{2}{*}{ Parameter } & \multicolumn{5}{|c|}{ Patients with CSU } & \multicolumn{5}{|c|}{ Control group } \\
\hline & $\mathrm{PB}^{*}$ & PC1 & PC2 & A1 & A2 & $\mathrm{PB}^{*}$ & PC1 & PC2 & A1 & $\mathrm{A} 2$ \\
\hline Basophil activity (\%) & 0.481 & 46.25 & 29.148 & 0.168 & 0.838 & 0.022 & 60.845 & 34.416 & 0.083 & 0.132 \\
\hline Mean \pm SD & \pm 0.539 & \pm 35.779 & \pm 24.483 & \pm 0.486 & \pm 2.378 & \pm 0.098 & \pm 26.197 & \pm 23.196 & \pm 0.176 & \pm 0.259 \\
\hline Minimum (\%) & 0.0 & 0.0 & 2.16 & 0.0 & 0.0 & 0.0 & 0.31 & 5.03 & 0.0 & 0.0 \\
\hline Maximum (\%) & 1.49 & 96.66 & 85.12 & 2.13 & 10.0 & 0.44 & 87.5 & 79.04 & 0.55 & 0.88 \\
\hline
\end{tabular}

SD - standard deviation, ${ }^{*} p=0.00468$

ticipants (mean age: 19 years; 14 females) without either allergy symptoms or CSU. Blood was collected into EDTA venipuncture tubes $(4 \mathrm{ml})$ and into coagulation activator tubes ( $4 \mathrm{ml}$ ) for each patient. Basophil activation as the presence of CD63 and CD63+CD203c at their surface was detected by flow cytometry test (Flow Cast Bühlmann Laboratories AG, No. FK-CCR and No. FK-CCR-203SET, respectively) following the manufacturer's recommendation. For each of the patients, $50 \mu \mathrm{l}$ whole blood sample was taken into tubes containing $50 \mu \mathrm{l}$ each: stimulation buffer (patient's background-PB), anti-FcERI (stimulating control 1 - PC1), fMLP (stimulating control 2 - PC2), patient's undiluted serum (allergen $1-\mathrm{A} 1$ ), patient's serum $1: 1$ isotonic solution (allergen 2-A2). Together with the cellular stimulation, a staining reagent was added containing a mixture of monoclonal antibodies to human CD63 (stimulation buffer with IL-3) or CD63+CD203C (stimulation buffer without IL-3). Erythrocytes were removed during lysing reaction. After a centrifugation step the cells were suspended in wash buffer and analysed by flow cytometry (FACScan, Becton Dickinson). Initially, basophil population was gated as those characterized by high CCR3 expression and low SSClow granularity. In the next step, expression of antigens CD63 and CD63+CD203c on basophils surface was used for analysis of their activity. In total population of basophils, percentage of cells activated by stimulus (positive control or sera) was identified (CellQuest, Becton Dickinson).

\section{Statistical analysis}

The data were analysed using Statistica Software Package, version 12.5 (Polish version; StatSoft, Poland). All tests were conducted from October 2018 to March 2019. All participants signed an informed consent form.
Laboratory tests were performed in the Laboratory at the Department of Internal Medicine, Pneumology and Allergology, Wroclaw Medical University, Poland. The study was approved by the Ethics Committee at Wroclaw Medical University, Poland (No. KB - 15/2018).

\section{Results}

In the non-stimulated negative sample (PB) the percentage of activated basophils was higher in patients with CSU (either CD63-positive or CD63+CD203c-positive) than in the control group and this difference was statistically significant $(p<0.05)$. Patients with CSU showed a significant difference $(p<0.05)$ in the basophil activation only for CD63 expression after stimulation with patient's serum 1: 1 isotonic solution (A2). No statistical differences were obtained in basophil activation between other nonspecific (PC1, PC2) or specific (A1, A2) stimulation. Obtained data are shown in Tables 1 and 2.

\section{Discussion}

CSU is a common skin disease characterized by recurrent, transitory, pruritic wheals with urticarial episodes being recorded during the time period of at least 6 weeks $[6,7]$. The basophil activation test is a reliable tool for monitoring cells activation [8, 9]. In our study we revealed that basophils from patients with CSU showed a primarily higher level of activation expressed as presence of CD63 and CD63+CD203 markers on the membrane. Moreover, incubation of donor basophils with serum leads to higher CD63 expression in the group with CSU than in controls. It might indicate that increased activation of basophils is associated with the greater predisposition to release mediators from these cells in 
patients with CSU. Our data are in accordance with another paper [10] reporting usefulness of CD63 expression in vitro for evaluation of basophil activation in patients with CSU during autoantibody stimulation. Interestingly, in our survey no statistical differences were obtained in basophil activation with sera for CD63+CD203c expression. Possible explanations are that basophils are known to express CD203c exclusively and CD203c expression should be evaluated in non-primed basophils [11]. Besides, presence of IL-3 may create false results in CD203c expression; to omit this bias, stimulation buffer without IL-3 was added together with a mixture of monoclonal antibodies to human CD63+CD203c. This study does have several limitations. Firstly, a small size of the investigated group was dependent on the preliminary schedule of our study. Secondly, the presented protocol to BAT included only two possible markers expressed; it would be interesting to assess a phenotyping panel with other receptors (e.g. CD123, CD164, CD193).

\section{Conclusions}

Our results of BAT in patients with CSU support the hypothesis about complex background of this disorder. Moreover, results indicate a greater degree of basophil activation in patients with CSU in remission than in the control group. Possible combination of BAT and other tests (e.g. autologous serum skin test, thyroid antibodies and anti-lgE level) might be useful for identification of patients with predominance of the autoimmune variant of CSU and typing patients responding (responders) and refractory (non-responders) to treatment with antihistamines. This is worth conducting a further investigation on a larger sample of patients.

\section{Acknowledgments}

This study was supported by grant No. ST. A200.17.042 from the Medical University in Wroclaw, Poland.

\section{Conflict of interest}

The authors declare no conflict of interest.

\section{References}

1. Zuberbier T, Henz BM, Fiebiger E, et al. Anti-fcepsilonrialpha serum autoantibodies in different subtypes of urticaria. Allergy 2000; 55: 951-4.

2. Panaszek B, Pawłowicz R, Grzegrzółka J, Obojski A. Autoreactive IgE in chronic spontaneous/idiopathic urticaria and basophil/mastocyte priming phenomenon, as a feature of autoimmune nature of the syndrome. Arch Immunol Ther Exp 2017; 65: 137-43.

3. Augey F, Gunera-Saad N, Bensaid B, et al. Chronic spontaneous urticaria is not an allergic disease. Eur I Dermatol 2011; 21: 349-53.
4. Dema B, Suzuki R, Rivera J. Rethinking the role of immunoglobulin $\mathrm{E}$ and its high-affinity receptor: new insights into allergy and beyond. Int Arch Allergy Immunol 2014; 164: 271-9.

5. Gaig P, García-Ortega P, Enrique E, Richart C. Successful treatment of chronic idiopathic urticaria associated with thyroid autoimmunity. J Investig Allergol Clin Immunol 2000; 10: 342-5.

6. Kikuchi Y, Kaplan A. Mechanisms of autoimmune activation of basophils in chronic urticaria. J Allergy Clin Immunol 2001; 107: 1056-62.

7. Gomutka K. Cold urticaria: a case report. Adv Derm Allergol 2012; 29: 60-2.

8. Boumiza R, Debard AL, Monneret G. The basophil activation test by flow cytometry: recent developments in clinical studies, standardization and emerging perspectives. Clin Mol Allergy 2005; 3: 9.

9. De Weck AL, Sanz ML, Gamboa PM, et al. Diagnostic tests based on human basophils: more potentials and perspectives than pitfalls. Int Arch Allergy Immunol 2008; 146: 177-89.

10. Gyimesi E, Sipka S, Danko K, et al. Basophil CD63 expression assay on highly sensitized atopic donor leukocytes - a useful method in chronic autoimmune urticaria. Br I Dermatol 2004; 151: 388-96.

11. Chirumbolo S. Basophil activation test for chronic urticaria. Ann Lab Med 2016; 36: 499-500. 